\title{
Role of c-Abl in the DNA damage stress response
}

\author{
Yosef SHAUL ${ }^{*}$, Merav BEN-YEHOYADA \\ Department of Molecular Genetics, Weizmann Institute of Scienc, Rehovot 76100, Israel
}

\begin{abstract}
c-Abl has been implicated in many cellular processes including differentiation, division, adhesion, death, and stress response. c-Abl is a latent tyrosine kinase that becomes activated in response to numerous extra- and intra-cellular stimuli. Here we briefly review the current knowledge about c-Abl involvement in the DNA-damage stress response and its implication on cell physiology.
\end{abstract}

Keywords: c-Abl, DNA-damage.

The c-Abl proto-oncoprotein is a member of the Src family of non-receptor tyrosine kinases. The highly evolutionarily conserved c-Abl was identified as the cellular homologue of the Ablelson murine leukemia virus and its transforming variants have been implicated in tumorigenesis and in many important cellular processes. c-Abl is ubiquitously expressed in cells and localizes both to the nucleus and the cytoplasm where it plays distinct roles. In recent years, many c-Abl protein-protein interactions have been resolved. However, the phenotypic outcomes of these interactions still need to be determined. A promising way to study c-Abl function is to identify its immediate downstream targets. This approach led to the identification of a number of candidates which by large belong to the DNA repair machinery [1].

\section{c-AbI STRUCTURE AND SUBCELLULAR LO- CALIZATION}

In mammalian cells c-Abl is ubiquitously expressed but with different sub-cellular localization depending on cell type. In fibroblasts it resides predominantly in the nucleus while in primary haematopoietic cells and neurons c-Abl is more cytoplasmic. In sharp contrast, all the transforming Abl variants are exclusively cytoplasmic. cAbl localization is controlled by NLS and NES motifs and it is thought to shuttle between the nucleus and cytoplasm depending on environmental signals [2]. In addition,

*Correspondence: Yosef SHAUL

Tel: +972-8-934-2320;

E-mail: yosef.shaul@weizmann.ac.il the human c-Abl gene encodes two isoforms, $1 \mathrm{a}$ and $1 \mathrm{~b}$ as a result of alternative splicing. While the 1a isoform is mostly cytoplasmic, the $1 \mathrm{~b}$ isoform is myristolated at the $\mathrm{N}$-terminus and localizes predominantly in the nucleus [3]. This pattern of cellular distribution hints at the possibility that c$\mathrm{Abl}$ is a shuttle kinase that transmits signaling between the major cellular compartments.

The c-Abl protein contains various defined domains. At the N-terminus, c-Abl has a Src homology 2 (SH2) domain that has high affinity for phosphorylated tyrosine residues and a Src homology 3 (SH3) domain that preferentially binds to proline-rich domains containing a PxxP motif. Search for c-Abl SH3 binding proteins proved fruitful in regard to defining its immediate downstream targets. Several proteins were identified, such as the Abi family, ATM, DNA-PK, BRCA1, and transcription factors p73 and RFX1 [1,4]. c-Abl is also characterized by its long $\mathrm{C}$-terminal tail containing nuclear localization (NLS) and nuclear export (NES) signals controling c-Abl sub-cellular localization. Also the tail contains a putative DNA-binding domain and F- and G-actin binding domains. With all of these structural domains, c-Abl is likely to simultaneously participate in many processes by direct protein-protein interactions.

\section{c-Abl KINASE ACTIVATION}

A key question is what is the mechanism that keeps the c-Abl kinase domain dormant. Originally, it was speculated that the c-Abl kinase domain is inhibited mostly by proteins bind to the c-Abl SH3 domain. Experiments performed with recombinant RFX1, an SH3 binding protein, and $\mathrm{c}-\mathrm{Abl}$ proved otherwise. This study revealed that $\mathrm{c}-$ 
Abl is innately inactive but becomes activated upon interaction with RFX1 [1]. It was proposed that similar to other Src kinases, the c-Abl kinase domain is under repression by an intra-molecular mechanism. Indeed, Pluk and coworkers showed that the inhibition of c-Abl activity is an intrinsic property and does not require a particular cellular inhibitor. In this model the c-Abl N-terminal "cap" structure (first 81 residues) that can fold over the SH3 domain plays the inhibitory role. This "cap" appears to bind at several regions across the molecule and stabilizes the regulated, inhibited conformation. These results were confirmed by resolving the c-Abl crystal structure. The autoinhibited c-Abl was shown to assemble through internal interactions between its $\mathrm{SH} 3$ and $\mathrm{SH} 2$ domains and the two lobes of the kinase domain [5]. However, these findings do not rule out the possibility that some of the c-Abl interacting proteins may help to stabilize the inactive conformation, while others help in its activation.

Various DNA damaging agents such as ionizing radiation (IR) activate the c-Abl kinase. IR mainly causes double strand breaks, but given the fact that the alkylating agents such as cisplatin and mitomycin C, DNA cross-linking agents, activate c-Abl as well, this suggests a robust cAbl response to DNA damage signaling. However, UV radiation does not trigger $\mathrm{c}-\mathrm{Abl}$ activation attributing certain specificity to the $\mathrm{c}-\mathrm{Abl}$ response. These findings set the stage for the search for upstream effectors of cAbl. An obvious candidate is the ataxia-telangiectasiamutated (ATM) protein. Atm is a nuclear member of a family of phosphatidylinositiol-3-kinase like $\left(\mathrm{PI}_{3} \mathrm{~K}\right)$ enzymes and binds the c-Abl SH3 domain [6]. When cells are exposed to IR, the ATM protein phosphorylates c-Abl at serine 465. This phosphorylation is responsible for c-Abl activation. AT-null cells are impaired in c-Abl kinase activation, attributing to ATM an important role in c-Abl activation in response to DNA damage stress. In addition, c-Abl kinase activation requires the mismatch repair (MMR) protein MLH1. In the MLH1-null colon cancer cell line HCT c-Abl kinase activation is impaired in response to cisplatin treatment and IR (unpublished), placing the MMR pathway up-stream to c-Abl activation. However, our new studies revealed that c-Abl is also activated by a second slow and gradual mechanism whose nature remains to be clarified.

\section{c-Abl-INDUCED APOPTOSIS}

Apoptosis is an essential process in the development of multi-cellular organisms, maintenance of tissue homeostasis and stress response. c-Abl null cells are impaired in the apoptotic response to IR [3] attributing a pro-apoptotic role to c-Abl. Consistent with this finding is the fact that cells stably expressing the c-Abl kinase-death mutant are resistant to IR-induced loss-of colonogenic-survival and apoptosis. The oncogenic c-Abl variants behave in a seemingly opposite manner to the wt c-Abl. In chronic myelogenous leukemia (CML) patients, the first exon of the cAbl tyrosine kinase is replaced by the $B C R$ gene. This translocation results in a constitutively active c-Abl kinase. $\mathrm{BCR} / \mathrm{ABL}$ expression results in enhanced proliferation, morphological transformation and anti-apoptotic effects.

The molecular basis of the pro-apoptotic role of c-Abl has not been yet completely resolved. It has been speculated that the tumor suppressor p53 is a downstream and immediate effector of c-Abl in this pathway. However, cAbl was shown to induce apoptosis regardless of p53 status (i.e. wt or null). p53-c-Abl direct interaction was detectable only in vitro. Moreover, p53 is not significantly tyrosine phosphorylated by c-Abl, even under over-expression conditions [7]. In contrast, p73, a p53 family member, proved to be a preferred candidate. p73 induces apoptosis in a variety of cell lines and supports transcription from promoters containing the p53-response element [8]. Over-production of p73 together with c-Abl is sufficient to induce apoptosis in fibroblasts. Unlike p53, p73 is efficiently tyrosine phosphorylated upon exposure to IR [9]. p73 contains a PxxP motif that interacts with the c-Abl SH3 domain. Furthermore, p73 is a direct substrate of c-Abl both in vitro and in vivo [8]. Upon induction of DNA-damage by cisplatin and IR p73 accumulates in a c-Abl dependent manner. Given the fact that the E3 ligase of p73 is not yet identified the molecular basis of p73 accumulation remaines a matter of speculation. It is very likely that the tyrosine phosphorylated $\mathrm{p} 73$ escapes the E3 ligase and proteasomal degradation. The fact that modified p73 translocates to the nuclear matrix lends further support to this model. Accumulated and modified p73 supports the transcription of the downstream proapoptotic genes [7].

\section{c-AbI IN DNA REPAIR PROCESSES}

DNA replication, recombination and repair, collectively termed DNA transactions, play a fundamental role in balancing between genomic stability and diversity. One possibility for c-Abl to get involved in DNA transaction processes is via its DNA binding domain. The c-Abl DNAbinding domain shows sequence similarity to $\mathrm{HMG}$ proteins. It also recognizes deformed DNA structures such as four-way junctions [1], like other HMG-domain proteins. Interestingly, HMG1 also specifically recognizes the DNA intra-strand crosslinks formed by the DNA-damaging agent cisplatin. c-Abl, therefore, might have the same activity, and may thus be recruited directly to cisplatin modified DNA regions. This attractive possibility, although not proven, might provide an alternative pathway for c-Abl 
activation by this genotoxic drug.

Many of the effectors, enzymes and auxiliary proteins that are involved in DNA transactions were shown to interact with and be modified by the c-Abl kinase. $\mathrm{c}-\mathrm{Abl}$ has been reported to interact with the homologous recombination protein Rad51 and inhibit in vitro its DNA strand exchange activity. Direct interaction was also demonstrated between c-Abl and DNA-PK. DNA-PK regulates nonhomologous end joining (NHEJ) repair processes. c-Abl phosphorylation of DNA-PK causes the dissociation of DNA-PK-Ku protein complex from the DNA and reduced NHEJ repair [3]. Tyrosine phosphorylation of the WRN protein inhibits its exonuclease and helicase activities. An additional player in the response mechanism to double strand breaks is BRCA1. In cells exposed to IR, c-Abl-BRCA1 interaction is interrupted [4]. Altogether, these data hint the possibility that $\mathrm{c}-\mathrm{Abl}$ regulates DNA repair. An interesting model is that $\mathrm{c}-\mathrm{Abl}$ is the decision maker, namely it is the role of $\mathrm{c}-\mathrm{Abl}$ to activate the proapoptotic pathway when the DNA damage is too severe to be repaired.

\section{REFERENCE}

1 Shaul Y. c-Abl: activation and nuclear targets. Cell Death Differ 2000; 7:10-6.

2 Taagepera S, McDonald D, Loeb JE, et al. Nuclear-cytoplasmic shuttling of C-ABL tyrosine kinase. Proc Natl Acad Sci USA 1998; 95:7457-62.

3 Kharbanda S, Yuan ZM, Weichselbaum R, et al. Functional role for the c-Abl protein tyrosine kinase in the cellular response to genotoxic stress. Biochim Biophys Acta 1997; 1333:O1-7.

4 Wang JY. Controlling Abl: auto-inhibition and co-inhibition? Nat Cell Biol 2004; 6:3-7.

5 Pluk H, Dorey K, Superti-Furga G. Autoinhibition of c-Abl. Cell 2002; 108:247-59.

6 Shiloh Y. ATM and related protein kinases: safeguarding genome integrity. Nat Rev Cancer 2003; 3:155-68.

7 Ben-Yehoyada M, Ben-Dor I, Shaul Y. c-Abl tyrosine kinase selectively regulates p 73 nuclear matrix association. J Biol Chem 2003; 278:34475-82.

8 White E, Prives C. DNA damage enables p73. Nature 1999; 399: 734-5, 737.

9 Agami R, Blandino G, Oren M, et al. Interaction of c-Abl and p73alpha and their collaboration to induce apoptosis. Nature 1999; 399:809-13. 